https://doi.org/10.48009/2_iis_2009_95-100

\title{
The Next Generation Internet Protocol, IPV6: An Overview
}

\author{
Mohamad Ladan, Haigazian University, Beirut LEBANON, mladan@haigazian.edu.lb
}

\begin{abstract}
The current Internet Protocol, IPv4, is the original standard Internet Protocol set up for handling IP addresses when the Internet was initial developed by DARPA (Defense Advanced Research Projects Agency) in the early 1970s. The IPv4 uses a 32 bit address field which provides for 4,294,967,296 unique Internet addresses. This number was deemed to be more than enough to last beyond any foreseeable requirements because in the early 1970's the population of the earth was less than 4 billion people and personal computers did not exist. Now, the rapid explosion of the Internet fueled by the vast number of personal computers attaching to it, made it clear that the IPv4 address space was already consumed to the point that a replacement had to be found. The next generation IP, IPv6, was developed in response to this situation. In this paper, I will try to give a complete overview of this new protocol. I start by giving a history of the internet and its current protocol. Then I will describe the main features of the new protocol, IPv6, and how they have solved the current limitation of the current protocol.
\end{abstract}

Keywords: Internet Protocol, Networks, IPv4, IPv6

\section{INTRODUCTION}

Internet Protocol, IP, is the network-layer protocol and one of the pillars which supports the Internet. A protocol is a set of rules which specifies how a packet of bits will be interpreted and how a node should react to the datagrams it receives. It defines the semantics of a packet, how the bits should be interpreted, but also what the node should do if something goes wrong. In general, the IP has the following main services:

- Addressing: This is the field of the destination of a packet in the packet header. While packet travel from root to root intermediate roots decide according to this address to which root to send the packet next.

- Fragmentation: Larger packets are divided into smaller ones and at the target the packets are recombined.
- Packet timeouts: In each IP packet there is a Time to Live field. Each time when a packet travel through a router, that router decrements the Time to Live number and forward the packet to the next router. When the number gets to zero the packet will be discarded.

- Type of Service. Each IP packet is labeled whit an abstract type of services and according to this label the prioritization of packet is defined.

The number of the Internet users is increasing more and more day after day, and it is realized that the lifetime of the IPv4 address space is limited. IPv4 uses 32-bit addresses, and with the growth of the Internet, these have become a scarce and valuable commodity. Organizations have gone to great lengths to deal with the shortage and high cost of IPv4 addresses. In 1991, the IETF decided that the IPv4 had outlived its design. The new version of IP, called either IPng (Next Generation) or IPv6 (version 6), was the result of a long and tumultuous process which came to a head in 1994, when the Internet Engineering Task Force, IETF gave a clear direction for IPv6. Since the new IPv6/IPng architecture solves the address space problem in an effective way, the need for the new version is increasing day after day. In its meeting in Brussels on 27/05/2008, The Commission of the European Communities has sent a communication to the European Parliament, the council, the European economic and social committee and the committee of the regions recommending a number of actions to achieve widespread IPv6 implementation in Europe by 2010 [10].

IPv6/IPng is a new version of IP which is designed to be an evolutionary step from IPv4. It is designed to solve the problems of IPv4 and to be a natural increment of it. It does so by creating a new version of the protocol which serves the function of IPv4, but without the same limitations of IPv4. The differences between IPv6 and IPv4 are in five major areas: addressing and routing, security, network address translation, administrative workload, and support for mobile devices. The rest of this paper is organized as 
follows: Section 2 describes the current internet protocol and the main problems with it. Section 3 covers an overview of the next generation protocol, IPV6, and discusses its main features and major goals. Finally, section 4 gives a conclusion and a summary.

\section{CURRENT INTERNET PROTOCOL}

The current Internet Protocol is called IPv4, it uses 32-bit address fields and has the header format shown in Figure 1.

Figure 1: IPV4 Header Format

\begin{tabular}{|c|c|c|c|c|}
\hline $\begin{array}{c}\text { Version } \\
4 \text { bits }\end{array}$ & $\begin{array}{c}\text { IHL } \\
4 \\
\text { bits }\end{array}$ & $\begin{array}{c}\text { Type of } \\
\text { Services } \\
8 \text { bits }\end{array}$ & \multicolumn{2}{|c|}{$\begin{array}{c}\text { Total Length } \\
16 \text { bits }\end{array}$} \\
\hline \multicolumn{3}{|c|}{$\begin{array}{c}\text { Identification } \\
16 \text { bits }\end{array}$} & $\begin{array}{c}\text { Flags } \\
3 \\
\text { bits }\end{array}$ & $\begin{array}{c}\text { Fragmentation } \\
\text { Offset } \\
13 \text { bits }\end{array}$ \\
\hline TTL & $\begin{array}{c}\text { Protocol } \\
8 \text { bits }\end{array}$ & $\begin{array}{c}\text { Header Checksum } \\
16 \text { bits }\end{array}$ \\
\hline bits & $\begin{array}{c}\text { Source Address } \\
32 \text { bits }\end{array}$ \\
\hline \multicolumn{3}{|c|}{ Destination Address } \\
32 bits \\
\hline \multicolumn{3}{|c|}{ Options } \\
\hline
\end{tabular}

IPv4 protocol organizes addressing by simply combining network number and host number. So, in the 32 bits address field the network and the host under that network is defined. There are three different kinds of addressing, which are Class A, Class B, and Class C. As each class of the address will support different number of network and hosts under networks, addresses are assigned to a network, according to the size of (hosts in the network) the network.

These classes differ in the number of nets and hosts as shown in Figure 2:

Figure 2: Three main classes of IPv4's addresses

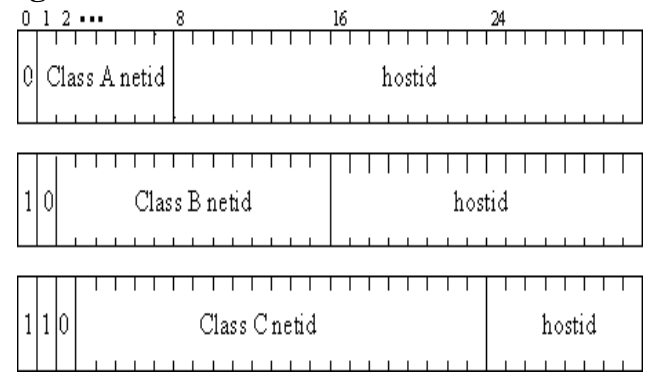

Class A address is the largest host supporting class. This class has a 7-bit network number, and a 24-bit host number. It can have up to 125 networks with 16 million hosts per network. All of these addresses are taken by the world's big organizations.

Class B addresses contains a 14-bit network number, and a 16-bit host number. The number of the networks which can have this class of addressing is 16,384 , and each network can contain 65,536 hosts.

Class $\mathrm{C}$ addresses are suitable for the small networks. This class has 21-bit of network number and 8-bit host address. This can contain up to 2 million networks with 256 hosts per network.

\section{Main Problems with the Current Internet Protocol \\ The main problems of the IPv4 can be} divided into two main classes of problems: Address problems that are related to the limited address space and address allocation, and Options problems that are related to options required by new applications development like QoS. In what follows, I will summarize the different problems in each class.

\section{Address problems:}

- Limited address space: IPv4 has a 32 bit address field which gives up to four billion addresses. Although this seems like a very large number of addresses, many experts believe that we are nearly out of these available addresses. The address space of IPv4 has been used up to a considerable extent. At the end of January 2008 about $16 \%$ was left in the IANA pool, i.e. approximately 700 million IPv4 addresses. There are widely quoted and regularly updated estimates which forecast the exhaustion of the unallocated IANA pool somewhere between 2010 and 2011 [10]. One main reason of this is because a significant number of organizations joining the Internet in the early days were granted multiple large blocks of class A addresses. This means that organization like Apple, HP, MIT, Stanford etc each have more address space than China.

- Inefficient allocation: Routing is only practical by separating large blocks of address space into networks. So the practical limit is a few hundred million addresses. In other words, the addresses are not efficiently assigned and used. For example, if an organization has a bit more hosts than Class C it will apply for Class B address and as a result of this 
the most of the host addresses will not be used by the organization. To solve this problem a new protocol named Common Interdomain Routing Protocol (CIDR). In this protocol instead of assigning a class $\mathrm{B}$ address to an organization it assign a block of Class B addresses. But still this is not enough to solve the problem of addressing.

- Routing table size: Routing table size is growing so fast has always been a problem. Using two hierarchies to define the number of connected hosts to internet resulted in a huge size routing table. So it takes more time to process the routing tables. In general the network speed has been increasing faster than router hardware, so extra performance goes into shovelling packets faster rather than handling bigger routing tables. It's not only an issue of routing table size, but also the dynamic nature of routing updates.

\section{Options related problems:}

- Provider selection: By this way somebody can choose special providers for routing the packet. In the commercial usage of internet this take an important role. For example while sending data one needs a trusted provider. This feature is not supported in IPv4. [3]

- Scalable multicast. In Ip protocols multicasting is possible only in subnets. In some situations like real time applications it can be possible to address different hosts in different subnets. [5]

- Mobility. "plug-and-play": As the mobility is the target of the IP development. The current version do not support mobile connection. Actually you have to configure manually. Instead of this there should be autoconfiguration to make mobility more efficient. This would mean that your host would have to get an address from a provider. [6]

- Real-time flow: If we look at the development of the Internet, the target is real time data transfer and Multimedia. So, the protocol should support this applications in an efficient way. For example
Video conferancing. In the current version the QoS is low. [6]

- Autoconfiguration: IPv4 do not support auto configuration. Making manually configuration of each host is time consuming and difficult. IPv6 requires that hosts and routers must support automatic configuration, it is not an option. Typically manager program the routers with their addresses and networks then simply plug a host onto a network. The host will talk to the network to get its IP address and its routes. It is even possible to have a network with no routers or servers and still communicate.

- Using of LANs: By using IPv4 on LANs there should be a correspondence between IPv4 and MAC. IPv4 provides this by using ARP that utilizes broadcast MAC layer transmissions [8]. On the other hand, in IPv6 this is done by using "neighbor discovery" method. This method is more efficient than ARP when used on LANs.

\section{NEXT GENERATION INTERNET PROTOCOL: IPVG}

\section{Overview of the IPV6}

The next generation Internet Protocol, called IPv4 or IPng, uses 128-bit address fields and has the header format shown in Figure 2.

Figure 2: IPv6 Header Format

\begin{tabular}{|l|l|l|l|}
\hline $\begin{array}{l}\text { Version } \\
4 \text { bits }\end{array}$ & $\begin{array}{l}\text { Priority } \\
4 \text { bits }\end{array}$ & \multicolumn{2}{|l|}{$\begin{array}{l}\text { Flow Label } \\
24 \text { bits }\end{array}$} \\
\hline $\begin{array}{l}\text { Payload Length } \\
16 \text { bits }\end{array}$ & $\begin{array}{l}\text { Next } \\
\text { Header } \\
8 \text { bits }\end{array}$ & $\begin{array}{l}\text { Hop } \\
\text { Limit } \\
8 \text { bits }\end{array}$ \\
\hline $\begin{array}{l}\text { Source Address } \\
128 \text { bits }\end{array}$ \\
\hline $\begin{array}{l}\text { Destination Address } \\
128 \text { bits }\end{array}$ \\
\hline
\end{tabular}

It is a new version of the Internet Protocol, designed to take an evolutionary step from IPv4. It was not a design goal to take a radical step away from IPv4. Functions which work in IPv4 were kept in IPV6. Functions which didn't work were removed. The 
most visible change in IPv6 is that addresses balloon from 32-bits to 128-bits. With such a huge address space, ISPs will have sufficient IP addresses to allocate enough addresses to every customer so that every IP device has a truly unique address. Additional address space will also help the core of the Internet by reducing the size and complexity of the global routing tables. Although IPv6 doesn't solve the problems of routing in the Internet, it can help in several areas, reducing the initial size of the tables and offering a hierarchical address space.

\section{Major goals of IPV6}

The major goals of IPV6 can be summarized as follows:

- $\quad$ Reduce the total time which people have to spend configuring and managing systems. An IPv6 system can participate in "stateless" autoconfiguration, where it creates a guaranteed-unique IP address by combining its LAN MAC address with a prefix provided by the network router [5]

- Speed up the network, both from a performance and from a deployment point of view. IPv6 embodies the lessons learned at trying to build high-speed routers for IPv4 by changing the header of the IP packet to be more regular and to streamline the work of high-speed routers moving packets across the Internet backbone. IPv6 has fixed header sizes and less number of fields. A side effect of the redesign of the IP packet header is that future extensions to IPv6 are simplified: adding a new option to IP can be done without a major re-engineering of IP routers everywhere.

- Provide multicast capabilities for highbandwidth multimedia and fault tolerance applications. Multimedia applications can take advantage of multicast: the transmission of a single datagram to multiple receivers. Although IPv4 has some multicast capabilities, these are optional and not every router and host supports them. With IPv6, multicast is a requirement. IPv6 also defines a new kind of service, called "anycast." Like multicast, anycast has groups of nodes which send and receive packets. But when a packet is sent to an anycast group in IPv6, it is only delivered to one of the members of the group. This new capability is especially appropriate in a fault-tolerant environment: web servers and
DNS servers could all benefit from IPv6's anycast technology.

- Required security protocols. Secure networks will be easier to build and deploy in an IPv6 because the new IPSec security protocols, ESP (encapsulating security protocol) and $\mathrm{AH}$ (authentication header) are built-in and required in IPV6 and not add-ons like in IPv4.

\section{Major changes from IPV4 to IPV6}

The changes from IPv4 to IPV6 can be primarily categorized as the following:

- Expanded Routing and Addressing Capabilities. IPV6 increases the IP address size from 32 bits to 128 bits, to support more levels of addressing hierarchy and a much greater number of addressable nodes, and simpler auto-configuration of addresses. IPV6 addresses are four times the number of bits as IPv4 addresses (128 vs. 32). This is 4 Billion times 4 Billion times 4 Billion $\left(2^{\wedge \wedge 96)}\right.$ times the size of the IPv4 address space $\left(2^{\wedge \wedge} 32\right)$. This works out to be: $340,282,366,920,938,463,463,374,607,431,7$ $68,211,456$. It may seem like overkill to have this many addresses available, However, many visionary individuals believe that eventually every wired and wireless computer, cell phone, PDA, household appliance, security camera, devices that haven't yet been invented, will each have their own unique Internet address [8]. These address are written as:

○ eight colon-separated 16bit hex values, $\quad$ eg fe80:0000:0000:0000:02 $00:$ eff:fe 4 e: 0000

- leading zeroes can be omitted from each 16bit value, fe80:0:0:0:200:e8ff:fe $4 e: 0$

- contiguous zero values can be replaced by ': :', fe8 $80: 200:$ eff:fe4e: 0

- A new type of address called a "anycast address" is defined, to identify sets of nodes where a packet sent to an anycast address is delivered to one of the nodes. The use of anycast addresses in the IPng source route allows nodes to control the path which their traffic flows.

- Header Format Simplification. Some IPv4 header fields have been dropped or made 
optional, to reduce the common-case processing cost of packet handling and to keep the bandwidth cost of the IPV6 header as low as possible despite the increased size of the addresses. Even though the IPV6 addresses are four time longer than the IPv4 addresses, the IPV6 header is only twice the size of the IPv4 header.

- Improved Support for Options. Changes in the way IP header options are encoded allows for more efficient forwarding, less stringent limits on the length of options, and greater flexibility for introducing new options in the future.

- Quality-of-Service Capabilities. A new capability is added to enable the labeling of packets belonging to particular traffic "flows" for which the sender requests special handling, such as non-default quality of service or "real- time" service.

- Authentication and Privacy Capabilities. IPV6 includes the definition of extensions which provide support for authentication, data integrity, and confidentiality.

\section{SUMMARY and CONCLUSION}

IPV6 provides a platform for new internet functionality that will be required in the near future. Enormous improvements have been made in IPv6 over IPv4. The first improvement is the extending of the address length from 32 bits to 128 bits, creating a huge number of available addresses. The Second major improvement of IPv6 is the simplification of the header. IPv6 contains only 7 fields as compared to 13 fields in IPv4. As a result, router can process packets faster, resulting in the improvement of throughput. The third improvement is the support for multicasting, IPv4 repeatedly routes multiple copies of data to each and every receiver, creating obvious congestion problems. IPv6 introduces an anycast address to help with this problem. Anycast identifies nodes that can share packets, and routers use that information to send just one set of data to service several nodes. The fourth improvement is the better support for security; IPv4 has trouble supporting security because an application can encode operations at only one length: 40 bytes. IPv6 permits encoding at variable lengths and at lengths greater than 40 bytes. Now applications can support authentication and security encapsulation. A final improvement is the support for autoconfiguration, this means that IPv6 hosts can plug into the network and start communicating without requiring special configuration, whether the connection is to isolated stand-alone networks or to a large corporate network.

IPV6 may take some time to be fully used and deployed. The factors that support this opinion include the following: IPV6 represents a straightforward evolution of IPv4. It provides huge address space that takes along long time to be exhausted. It is already supported by major Operating Systems. And since, as with an operating system upgrade, users must only learn the new features, relying upon the fact that many of the reasons they chose the operating system in the first place are still present. IPv6 offers users a number of new, or restored, features that will enhance the Internet for decades to come. This fact will make the migration and replacement phase much less painful, and costly, than if IPv6 were an entirely new and dissimilar protocol.

\section{REFERENCES}

1. S. Deering, B. Haberman, B. Zill, IP Version 6 Scoped Address Architecture, Internet Draft, draft-ietf-ipngwg-scopingarch-02.txt, March 2001.

2. R. Draves, Default Address Selection for IPv6 , Internet Draft, June 2001: http://www.ietf.org/internet-drafts/draft-ietfipngwg-default-addr-select-05.txt

3. 3Com Technology, IPv6: Next Generation Internet $\quad$ Protocol, 11.8.1999 http://www.3com.com/nsc/ipv6.html

4. A. Tanenbaum. Computer Networks, Fourth Edition. Pren. Hall. 2004.

5. Commer, E. Douglas. Internetworking with TCP/IP.Third Edition, Volume I Prentice Hall International 1995.

6. IPv6: The New Internet Protocol. Stallings. W. IEEE Communications Magazine, Volume: 347, July 1996, Pages: 96-108

7. California Institute of Technology (Center for Advanced Computing Research), Internet Protocol Next Generation (IPv6), 24 Apr 1998 http://www.cacr.caltech.edu/ rfire/ipv6/tuto rial.html

8. The next generation of the Internet: Aspects of the Internet Protocol Version 6. Lee, D.C.; Lough, D.L.; Midkiff, S.F.; Davis, 
N.J., IV; Benchoff, P.E. IEEE Network Volume: 12 1, Jan-Feb 1998, Pages: 28-33

9. Blanchet, Marc \& Parent, Florent, IPv6 Primer, 05.04.1999: http://www.viagenie.qc.ca/en/ipv6/tutorialipv6-ripe33/html/index.htm

10. The Commission of the European Communities communication to the
European Parliament, the council, the European economic and social committee and the committee of the regions. Brussels, $27 / 05 / 2008$

http://ec.europa.eu/information_society/poli cy/ipv6/docs/european_day/communication final_27052008_en.pdf 